\title{
WHITE TRASH - DAS RASSIFIZIERTE „PREKARIAT“ IM POSTKOLONIALEN DEUTSCHLAND
}

ESKE WOLLRAD

\begin{abstract}
„Durch welches Ausschließungssystem, durch wessen Ausmerzung, durch die Ziehung welcher Scheidelinie, durch welches Spiel von Negation und Ausgrenzung kann eine Gesellschaft beginnen zu funktionieren?"
\end{abstract}

(Foucault 1976: 57)

Ausschließungssysteme bilden notwendige Voraussetzungen für das Funktionieren einer Gesellschaft und arbeiten mit Dichotomisierungen: oben - unten, reich - arm, männlich - weiblich, Weiß - Schwarz, legal illegal, gesund - krank (Foucault 1976: 57). Ein Element dieser dichotomen Konstruktion repräsentiert jeweils die Norm: männlich, Weiß, gesund usw. Dieser Beitrag thematisiert den spezifischen Zusammenhang von Unterschicht und Weißsein und seine Repräsentation im bundesdeutschen Kontext als White trash. Den Referenzrahmen der Untersuchung bildet postkoloniale feministische Kritik, die voraussetzt, dass koloniale Wissensproduktion nach 1918 nicht einfach aufhörte und vergessen wurde, sondern dass gegenwärtige Diskurse vielfach Echos dieser Wissensproduktion enthalten. Dieser Beitrag spürt einige dieser Echos auf und skizziert, wie gegenwärtige Diskurse zum Prekariat Prozesse von Rassifizierung rekapitulieren und revitalisieren. 


\section{White trash in gegenwärtigen Debatten}

Der Begriff White trash stammt ursprünglich aus den USA und wurde seit dem frühen 19. Jahrhundert auf bestimmte arme Weiße angewendet. Dabei war - und ist - der „Weiße Abfall“ nicht nur die Definition einer ökonomischen Kondition, sondern ebenso die Bezeichnung einer spezifischen stereotypisierten trash Kultur, die geprägt ist durch eine vermeintlich gefährliche und exzessive Sexualität und durch extremen Rassismus (ebd.: 171). Trash bezeichnet nicht nur den Aspekt des Im-MüllLebens, sondern auch den des Müll-Seins: White trash trägt die Signatur moralischer Verkommenheit - White trash ist Abschaum. In den USamerikanischen Medien der 1980er und 1990er Jahre wurde White trash Mainstream: Fernsehserien wie Roseanne oder Grace under Fire waren außerordentlich erfolgreich, und heute ist es der Rapper Eminem, der White trash Kultstatus verleiht."

Seit Ende der 1990er Jahre befassen sich die Critical Whiteness Studies mit White trash, denn anders als das normative Whiteness, das seine Macht gerade aus der Unsichtbarkeit und aus der Unbenanntheit bezieht, ist White trash die einzige Gruppe der Weißen, die im dominanten Diskurs als Weiß benannt wird. Insbesondere für die dekonstruktivistisch ausgerichtete Richtung der Critical Whiteness Studies beweist die Existenz des White trash, dass es eine einheitliche privilegierte und machtvolle Weiße Identität nicht gibt (vgl. Newitz/Wray 1997: 168). Die Pluralisierung von Weißsein kann jedoch eine herrschaftsaffirmative Komponente beinhalten, wenn das Weißsein des White trash in der Betrachtung fast verschwindet, was eine Gleichsetzung des White trash mit der Situation armer Schwarzer nahe legt. Diese Gefahr der Trivialisierung rassistischer Ausschließungssysteme ist auch für bundesdeutsche Weißseinsforschung bedenkenswert.

Zunehmend begegnet der Begriff White trash in bundesdeutschen Diskursen, insbesondere in den Medien, und bezieht sich meist auf eine Gruppe, die seit einiger Zeit als „Prekariat“ definiert wird. Die Debatte um den Begriff und darum, was oder wen er eigentlich bezeichnet, intensivierte sich durch die Studie der Friedrich-Ebert-Stiftung „Gesellschaft im Reformprozess“, publiziert 2006 (vgl. Müller-Hilmer 2006): sie verwendete den Begriff ,abgehängtes Prekariat“. Bei dem Begriff „Prekariat“ handelt es sich um einen Neologismus, abgeleitet vom Adjektiv „prekär“ (bedenklich, misslich). Das ,abgehängte Prekariat“ - so die Studie - ist geprägt von sozialem Ausschluss und Abstiegserfahrungen, von einem sehr hohen Männeranteil, dem stärksten „Osttyp“ (ein Begriff, den die Studie nicht näher definiert), größter finanzieller Unsicherheit und dem größten Rechtswählerpotential. Diese Gruppe 
favorisiert die Abschottung gegenüber Ausländern und reagiert auf Verunsicherung mit ausgeprägtem Ethnozentrismus (ebd.: 85). Menschen dieser Gruppe empfinden ihre gesamte Lebenssituation als ausgesprochen prekär. Die FES-Studie fügt ,,abgehängt“ hinzu. Dies evoziert Bilder von den Verlorenen, Überflüssigen, Überzähligen, Überschüssigen, Nutzlosen. Vor allem verbindet sich dieser Begriff mit Vorstellungen von Unumkehrbarkeit - der Zug ist abgefahren, die Abgehängten bleiben auf der Strecke stehen.

Imaginationen des Prekariats sind vielfach skandalisiert worden auch in der Wissenschaft. So spricht der Historiker Paul Nolte von der neuen Unterschicht, in der sich gleichgültige und verkommene Lebensarten ausgebildet haben, in denen „Vernachlässigung, Verwahrlosung, im Extremfall Gewalt“" (Nolte 2006: 99) herrschen. Was diese neue Unterschicht kennzeichnet, ist nach Nolte vor allem das Defizitäre: Ernährungsdefizite, Bewegungsmangel, Sprachdefizite und Bildungsrückstände, dagegen besteht ein Übermaß an Fernseh- und Handykonsum (vgl. Kessel 2005), wobei diese Gruppe das sog. „Unterschichtsfernsehen" (RTL und SAT.1) (Nolte 2004: 42) favorisiert.

Diese Defizite werden diskursiv mit moralisch ethischen Defiziten verkoppelt, anschaulich verdichtet auch in den Medien. Im Heft GEO Wissen 2005 zum Thema „Sünde und Moral“ werden u. a. die sieben Todsünden in einer Fotostrecke thematisiert: Die vierte Todsünde wird „soziale Verwahrlosung“' genannt:

„Soziale Verwahrlosung - diese Trägheit des Herzens - gibt es in allen Gesellschaftsschichten. Auffällig wird sie aber nur dort, wo sich innere Lieblosigkeit und äußere Wurstigkeit vereinen. Im so genannten white trash, der heruntergekommenen Lebensart eines Teils der Unterschicht, ergeben sie eine unansehnliche Melange: Menschen, die seelisch erkalten und unempfänglich werden für Signale jeglicher Art.“ (GEO Wissen 2005).

Das Bild zeigt eine Weiße Frau und einen Weißen Mann, die auf einem Sofa eine unansehnliche Mahlzeit zu sich nehmen. Beide sind leicht bekleidet, ihre Kleidung weist Flecken auf, und die Frau ist deutlich übergewichtig. Beide starren vor sich hin, zwischen ihnen sitzen ein Hund und eine Katze. Diese Bebilderung inszeniert nicht Armut, sondern „Verkommenheit“" und Schmutz, nicht Hunger, sondern abstossende Sättigung, welche in Fettleibigkeit mündet. Der White trash wird als Abschaum der Gesellschaft imaginiert, als wertloser Weißer Abfall, weil es ihm am Menschlichen, an Seele und an der Fähigkeit zu empfinden mangelt. 


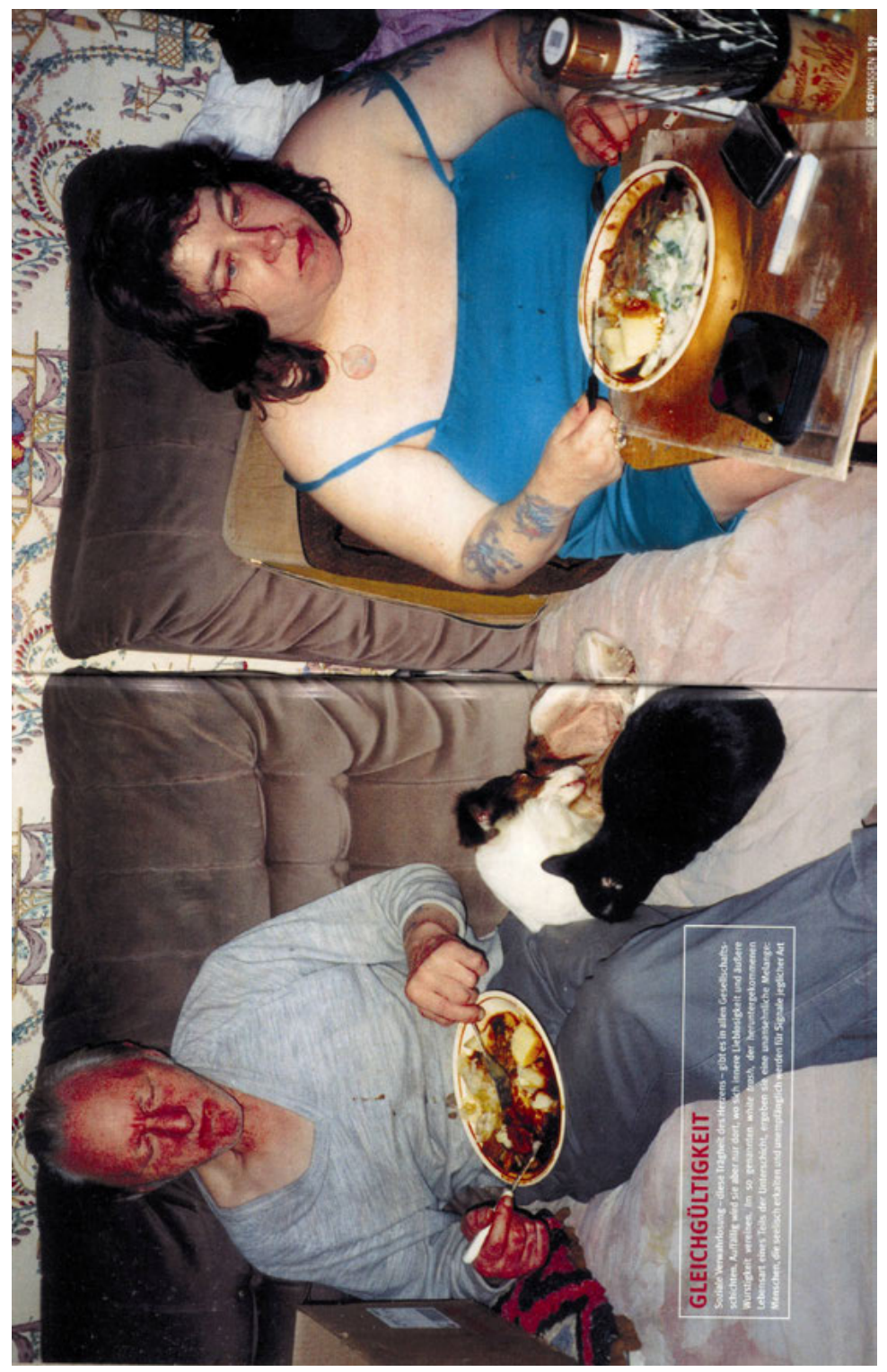

Abb. 1: Foto aus GEO Wissen 2005, Nr. 35. 
Dieser „Abfall“ ist nicht aus ökonomischen Gründen abgehängt, sondern verloren für die Gesellschaft, weil er seelisch nicht mehr erreichbar ist. Gleichzeitig ist er trash, weil er trash isst. Die GEO-Bebilderung stellt eine unmittelbare Verbindung von White trash mit unkontrollierter Aufnahme ungesunder Nahrung her, die zu Fettleibigkeit führt, welche den Staat jährlich 70 Milliarden Euro für die Behandlung ernährungsmittelbedingter Erkrankungen kostet. Im Kern revitalisiert dieses Bild das tief verankerte Stereotyp des Parasiten. So kritisiert der Bericht des Bundesministeriums für Wirtschaft und Arbeit vom August 2005 die angebliche Unterschichtsmentalität von Missbrauch, Abzocke und Selbstbedienung im Sozialstaat. Darin heißt es: „Biologen verwenden für ,Organismen', die zeitweise oder dauerhaft zur Befriedigung ihrer Nahrungsbedingungen auf Kosten anderer Lebewesen - ihren Wirten - leben', übereinstimmend die Bezeichnung ,Parasiten‘. Natürlich ist es völlig unstatthaft, Begriffe aus dem Tierreich auf Menschen zu übertragen. Schließlich ist Sozialbetrug nicht durch die Natur bestimmt, sondern vom Willen des Einzelnen gesteuert. “l Diese parasitären Weißen sind von einer Transfermentalität geprägt, d. h. sie erwarten, dass der Staat ihnen alle notwendigen materiellen Ressourcen selbstverständlich zur Verfügung stellt. Diese Mentalität - so Paul Nolte - ist nicht nur schädlich, sondern auch ansteckend: „Die Transfermentalität [...] hat sich in Jahrzehnten bis weit in die Mittelschichten vorgefressen." (Nolte 2004: 143).

Der White trash verkörpert das Gegenteil vom dominanten Weißsein: Dieses definiert sich über Macht, Zugang, Vernunft und Kontrolle im Sinne von Maß-Halten-Können. Während im hiesigen Kontext Forschungen zu dominantem Weißsein existieren (vgl. Wollrad 2005; Eggers et al. 2005), sind Studien zur Verhältnisbestimmung von dominantem Weißsein und dem White trash in Deutschland bislang nicht vorgelegt worden, was auch damit zu tun hat, dass die akademische Weißseinsforschung eine noch sehr junge Disziplin ist. Darüber spielen in gegenwärtigen Forschungen zur bundesdeutschen Klassengesellschaft Rassifizierungsdynamiken kaum eine Rolle. ${ }^{2}$ Postkoloniale Kritik hingegen behauptet, dass „Rasse“ keineswegs out ist, und dass gegenwärtige Diskurse zum Prekariat als Signatur von Parasitentum, Betrug, moralischer Verwahrlosung, seelischer Verrohung, Unreinheit und Krankheit Echos kolonialer Wissens- und Wahrheitsproduktion enthalten und dass diese der Ausgrenzung derer, die als „Prekarier“ bezeichnet werden, eine vertiefte Plausibilität verleihen.

1 Bericht des Bundesministeriums für Wirtschaft und Arbeit (2005: 10).

2 So beispielsweise der Politikwissenschaftler Albert Scharenberg: „Und es gibt sie doch: die Unterschicht“. In: Frankfurter Rundschau, 29.01.2007. 


\section{Zur Geschichte des White trash in Deutschland}

,[...] Diebe und Verbrecher aller Art, von den Abfällen der Gesellschaft lebend, Leute ohne bestimmten Arbeitszweig, Herumtreiber, dunkle Existenzen $[\ldots]^{\text {“ }}$

(Marx, MEW 7: 26)

Den White trash, diese dunklen Existenzen, kannte schon Karl Marx. Er nannte sie das Lumpenproletariat, „dieser Auswurf, Abfall, Abhub aller Klassen“ (Marx, 18. Brumaire, MEW 8: 160f.), „diese passive Verfaulung der untersten Schichten der alten Gesellschaft", die für den Klassenkampf nutzlos sind. „Dies Gesindel ist absolut käuflich. Wenn die französischen Arbeiter bei jeder Revolution an die Häuser schrieben: Mort aux voleurs! Tod den Dieben! Und auch manche erschossen, so geschah dies [...] in der richtigen Erkenntnis, dass man vor allem sich diese Bande vom Hals halten müsse." (Marx, Kommunistisches Manifest, MEW 4: 472) Für Marx gehörte zu den schmarotzenden Schichten nicht nur die Funktionärsklasse, sondern ebenso das Lumpenproletariat.

Marx Kritik an kapitalistischen Strukturen und das damit verbundene Heraufziehen gesellschaftlicher Umbrüche bekämpfte die Weiße Bourgeoisie mit der wirkungsvollsten Waffe des 19. Jahrhunderts: „Rasse“. Über die Biologisierung gesellschaftlicher Hierarchien suchte das Weiße Bürgertum seinen Herrschaftsanspruch abzusichern, indem es „Rasse“ als Inbegriff des gesunden Gesellschaftskörpers als Zellverband nicht degenerierter Individuen definierte, welcher gegen den schädlichen Einfluss parasitärer Fremdkörper verteidigt werden musste. Zentrale Begriffe waren Gesundheit und Degeneration: Der gesunde Gesellschaftskörper galt als Ergebnis rassenhygienischer Maßnahmen und unauflöslich verbunden mit Heterosexualität, ehelichen Geschlechterbeziehungen und fortpflanzungsorientierter Sexualität. Degeneration hingegen gefährdete den Fortbestand dieses „Volkskörpers“.

Sieben Jahre nach Erscheinen des Kommunistischen Manifests erschien 1855 das Werk „Die Ungleichheit der Menschenrassen“ von Arthur de Gobineau. Er führte soziale Schichtenbildung auf „Rassenunterschiede“ zurück und behauptete, die Folge von „Rassenmischung“ sei der Untergang jeder Zivilisation. Seine Theorie der „Rassen“ und der „Rassendegeneration“ wurde vielfach rezipiert - auch in Deutschland, intensiviert seit Beginn des deutschen Kolonialismus. Der Medizinhistoriker Pascal Grosse schreibt: „Die wirkungsmächtigste Idee [...], die der deutsche Kolonialismus hervorbrachte, war das Konstrukt der rassisch reinen biologischen Reproduktion als neue Grundlage des deutschen 
Nationalstaates.“ (Grosse 2000: 240). Dieses Konstrukt von „Rassereinheit“" war also keine Erfindung des Nationalsozialismus, sondern „Rasse“ war seit Beginn des deutschen Kolonialismus eine zentrale Ordnungskategorie sozialer Beziehungen.

Da die Konstruktion von Rassehierarchien die Existenz von Klassen naturalisierte und Ausbeutungsverhältnisse somit $\mathrm{zu}$ verewigen suchte, hätten sozialistische Kreise die Kategorie „Rasse“ entschieden ablehnen müssen. Dies war aber nicht notwendig der Fall. So hatte die Sozialistin Oda Olberg keinen Zweifel daran, dass ,die Forderungen der Rassenhygiene in der sozialistischen Bewegung ihren wirksamsten Bahnbrecher“ (Olberg 1907, zit. n. Hund 1999: 103) hätten. Ihrem Genossen Karl Valentin Müller zufolge sind Weiße Arbeiter „Teil der Herrscherschicht dieser Erde“ und „Teil einer Rasse höherer Anlage“ (Müller 1925, zit. n. Hund 1999: 107). Andere Weiße teilten Olberg zufolge dieses Privileg nicht: ,[D]ie am meisten verelendeten Schichten des industriellen Proletariats [...] [sind] für die Rasse unrettbar verloren und zum Aussterben bestimmt." (Olberg 1907, zit. n. Hund 1999: 103) Nach Olberg ist das Lumpenproletariat „überhaupt keine wirtschaftliche, sondern eine biologische Kategorie, die sich aus dem Abfall aller sozialen Schichten bil-

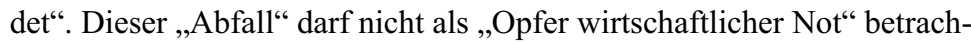
tet werden, sondern es handelt sich bei ihnen um „Entartete, die schon mit der Anwartschaft auf sozialen Schiffbruch geboren wurden“. Sie schloss „Abortus von Amts wegen“ nicht aus, hoffte auf die Möglichkeit verantwortungsvoller Euthanasie (Olberg 1926, zit. n. Hund 1999: 104f). Der Rassismusforscher Wulf Hund fasst zusammen: „Sozialistische Eugenik war der Versuch, die angeblich gesunden und hochwertigen Teile der Arbeiterklasse dadurch [der] vermeintlichen Einheit [der Rasse] zuzurechnen, dass ihr gegenüber eine Gruppe von Minderwertigen und Untermenschen erst konstituiert, dann denunziert und schließlich zur Ausmerzung bestimmt wurde.“ (Hund 1999: 108)

Auch Teile der bürgerlichen Frauenbewegung griffen die Idee der „Rassenhygiene“ begeistert auf. Helene Stöcker schrieb 1907: „Eine [...] menschliche Zuchtwahl, [...], die Eugenik, [...], die Züchtung eine[s] ,Übermenschen', [...] das ist das Ziel, das uns alle eint.“ (Stöcker 1911, zit. n. Bublitz 2000: 312) Attraktiv war Eugenik ihres Erachtens gerade deswegen, weil sie einer bestimmten spezifisch weiblichen Arbeit eine herausragende Bedeutung zuerkannte: der Gebärarbeit. Weißen Frauen des Bürgertums als „Bannträgerinnen des sittlichen Fortschritts“ (Fürth, zit. n. Bublitz 2000: 308) oblag nun die ehrenvolle Aufgabe, die Reinheit der „Rasse“ zu gewährleisten und Weiße Arbeiterfrauen zu Zucht und Sitte anzuhalten. Zucht ist hier wortwörtlich zu verstehen: Weiße Frauen des Bürgertums sollten sich vermehrt fortpflanzen und Weiße 
Frauen der Arbeiterklasse die Fortpflanzung begrenzen. Bei anderen sollte die Fortpflanzung gänzlich unterbunden werden. Helene Stöcker (1907): „Man wird [...] auch Mittel finden müssen, um unheilbar Kranke oder Entartete ganz an der Fortpflanzung zu verhindern.“ (zit. n. Bublitz 2000: 299)

Das koloniale Deutschland hat nicht nur „Rasse“ als zentrale Ordnungskategorie, sondern auch ein normatives Weißsein etabliert, dass bestimmte Weiße ausschloss. Diese wurden als eine Gruppe konstruiert, die als Weiß, aber dennoch als für die „Rasse“ verloren galt. Das Weiße Proletariat sollte sich gewiss sein, trotz des niederen Klassenstatus über Privilegien als Weiße zu verfügen und nicht auf der Rangstufe des Weißen Abfalls zu stehen. Gleichzeitig jedoch war die Abgrenzung gegen den White trash trotz aller Beteuerungen von der Höherwertigkeit der eigenen „Rasse“ nicht so einfach - das Szenario des Herabsinkens, der Degeneration, des Verfalls in die Gruppe der Verlorenen stand allen bedrohlich real vor Augen.

Der koloniale Terminus Technicus für diese Art des Verfalls war „Verkafferung“ und bedeutet so viel wie „Verwilderung“. „,,Verkafferte Weiße‘ waren arm, galten als moralisch verworfen, wären nicht mehr in eine europäische Gesellschaft reintegrierbar und daher machtpolitisch für die Nation nutzlos.“ (Grosse 2000: 85). Sie waren nicht nur nutzlos, sondern trugen auch in besonderer Weise zur „rassischen“ Degeneration bei, weil viele von ihnen die aus eugenischer Sicht größte Todsünde begangen: die Überschreitung der Rassengrenze. Diese „verblassten Weißen" hatten in den deutschen Kolonialgebieten oft ständigen Umgang mit der einheimischen Bevölkerung, und manche gingen Liebesbeziehungen mit Schwarzen ein. Gemäß der kolonialrassistischen Vorstellung wurde der „Verkafferte“ „,durch die Überschreitung der Grenze zu Afrikanern [...] als ,verlorenes Glied' für seine ,Rasse‘ betrachtet und aus der ,weißen Bevölkerung' exkommuniziert." (Schmidt-Lauber 1998: 378)

Echos von Imaginationen des Verkafferns sind auch heute noch hörbar. 


\section{White trash und die rassifizierten "Anderen“}

Die in der FES-Studie befragten Personen sind „natürlich“ Weiße Deutsche - andernfalls wäre dies explizit benannt worden. Die sich anschließenden Debatten schienen sich wie selbstverständlich nicht auf die Gruppe zu beziehen, die von vornherein für prekäre Arbeitsverhältnisse vorgesehen war, die schon immer befristete und ungesicherte Minijobs ausübte, und deren Erwerbsarbeitswelt als modernes Tagelöhnerwesen beschrieben werden kann. EinwanderInnen, Menschen mit Migrationshintergrund und illegalisiert in Deutschland Lebende sind meist konfrontiert mit einer äußerst prekären Lebenssituation: Erwerbslosigkeit oder ungeschützte Beschäftigungsverhältnisse, gekoppelt mit den schlechtesten Bildungschancen und Aufstiegsmöglichkeiten. Der kulturalistische Rassismus, der sie aufgrund ihrer kulturellen Herkunft und Sprache diskriminiert, zeichnet ein Bild von integrationsunwilligen islamischen Fundamentalisten, unterdrückten kopftuchtragenden Frauen, die sich in bedrohlichen Parallelgesellschaften eingerichtet haben - vorzugsweise in Berlin Neukölln. Doch entgegen der Proklamation ethnischer Segregation leben in Neukölln auch Weiße Deutsche, unter anderem der White trash. $\mathrm{Zu}$ Recht weist der Schriftsteller Feridun Zaimoglu darauf hin, „,dass es neben diesem Ethno-Proletariat [türkische Jugendliche in Neukölln] auch den White Trash gibt, das einheimische Prekariat. In diesem Milieu stellt sich genauso die Frage, wie integriert sind diese Leute eigentlich?"“(FR, 02.05.2007).

Begriffe, die Grundbestandteile des so genannten „Ausländerdiskurses“ sind, schimmern in Auseinandersetzungen mit dem Weißen Prekariat durch. Eine Tagung zum Prekariat, die im Mai 2007 in Jena stattfand, warf unter anderem die Frage auf: „Entstehen tatsächlich ,Parallel'- oder ,Subgesellschaften“?" Gleichzeitig leben in der ausländischen Parallelgesellschaft InländerInnen, die sich in diese Gesellschaft integriert haben. Im August 2005 berichtete eine ZDF-Reportage über eine Weiße Deutsche, die mit einem Türken verheiratet ist und gern türkisch spricht. In ihrem Viertel, so der Bericht, gibt es kaum noch deutsche Läden. „Um hier zurecht zu kommen, passen sich die Deutschen im eigenen Land an.“ (zit. n. Kessel 2005: 13)

Skandalisierende Bebilderungen des abgehängten Prekariats speisen sich somit aus zutiefst rassifizierten Diskursen über Parallelgesellschaften, Integration und Abschottung. Die Kluft, die diese Diskurse vertiefen, ist nicht die zwischen dem „Ethno-Proletariat" und dem White trash, sondern zwischen dem White trash und den wirklich Weißen, den wahren „Opfern“ der Prekarisierung. 


\section{Die Prekarisierung - zur Konstruktion der „wahren Opfer“}

Der Begriff der „Prekarisierung“ ist von dem des „Prekariats“ deutlich zu unterscheiden. (Nicht nur) in den Medien bezeichnet „Prekarisierung“ den Abstieg der Weißen Mittelschicht in prekäre Lebensverhältnisse, deren bislang als selbstverständlich vorausgesetzte materielle Privilegierung scheinbar erodiert. Die bereits erwähnte Tagung zum Prekariat in Jena fragte unter anderem: „Ist die Prekarisierung bei gebildeten, gut qualifizierten Gruppen angekommen? [...] Kann von einer ,Erosion der Mitte' gesprochen werden?“. Die so genannte prekarisierte Mitte - von der ZEIT als Gruppe der „Lumpenintelligenzler“ bezeichnet - wird als eine neue Klasse der Ausgebeuteten inszeniert. Zu ihnen zählen beispielsweise Hochschulabsolventinnen und -absolventen, die sich - gut ausgebildet, hochmotiviert und erwerbslos - von einem Praktikum zum nächsten hangeln: die „Generation Praktikum“. Die hoffnungsvollen AbsolventInnen sehen sich ungerecht behandelt von einer Gesellschaft, die ihnen einen schnellen Berufseinstieg verwehrt. Tatsächlich jedoch handelt es sich um eine gefühlte Benachteiligung, die nichts mit faktischem Abstieg und Privilegienverlust zu tun hat, sondern vielmehr Ausdruck einer spezifischen Anspruchshaltung einer Weißen Elite ist. Die Wirtschaftswissenschaftlerin Cordula Tutt schreibt:

„Die Vertreter der Generation ,Praktikum‘ gehören [...] eben nicht zu den ungerecht Behandelten oder zu jenen, die inzwischen oft als ,Prekariat', als die neue Klasse der ,ungeschützten Arbeiter' und Opfer des Schrumpfungsprozesses, bezeichnet werden. Viele von ihnen verdecken mit Praktika und schlecht bezahlter Arbeit nicht drohende Arbeitslosigkeit, sondern überbrükken mit prestigeträchtigen Stationen im Lebenslauf eine Phase, die sonst einen Sachbearbeiterjob in der Verwaltung, eine Anstellung in einem Provinzverlag oder das Anheuern in einem Callcenter bedeuten würde. Prekär ist die Lage unter Umständen eher für junge Menschen, die nicht von den Eltern unterstützt und finanziert werden und die deshalb keine Qualifizierungsschleifen drehen können.“ (FR, 23.03.2007; vgl. auch Tutt 2007).

Tutts Einschätzung lässt sich durch die Studie des DGB Jugend und der Hans Böckler Stiftung vom Februar 2007 untermauern. Die Studie heißt: „Generation Praktikum? Prekäre Beschäftigungsformen von Hochschulabsolventinnen und -absolventen“ (DGB Jugend 2007). Ihr zufolge machen $37 \%$ aller Hochschulabsolventinnen und -absolventen nach dem Abschluss mindestens ein Praktikum, dabei Frauen deutlich mehr (44\%) als Männer (23\%). 63\% der Praktika sind bezahlte Praktika, allerdings gering vergütet mit einem signifikanten geschlechtsspezifischen Unter- 
schied: Frauen verdienen durchschnittlich $543 €$, Männer hingegen 741 €. 37\% der PraktikantInnen erhalten keine Vergütung. Dies bedeutet: Zur Absolvierung eines Praktikum bedarf es finanzieller Ressourcen. Laut der Studie greifen zwei Drittel der PraktikantInnen bei bezahlten wie unbezahlten Praktika finanziell auf ihre Eltern zurück. Somit bezieht sich die „Generation Praktikum“ auf eine privilegierte Gruppe, die hohe Erwartungen an den Berufseinstieg hegt und es sich leisten kann, prestigeträchtige Praktika zu absolvieren. Darüber hinaus fand die Studie heraus, dass ein Jahr nach Ende des Studiums 93\% der AbsolventInnen eine erste Beschäftigung gefunden bzw. sich selbstständig gemacht haben oder freiberuflich tätig sind.

Mediale Inszenierungen von angeblich verarmenden Mittelschichtsangehörigen negieren, wo die Armut tatsächlich ansteigt: Einem Bericht des deutschen Instituts für Wirtschaftsforschung zufolge wächst sie von unten nach oben im Sinne einer zunehmenden Kumulation materieller Benachteiligungen, und nicht in Gestalt eines von der Mitte her immer mehr bröckelnden Wohlstands (FR, 22.03.2007). Die phantasmatischen Abstiegsszenarien der Mitte sind m. E. als Krisenphänomen zu deuten: In die Krise geraten ist nicht das ökonomische Gefälle und die Chancen der Weißen Mittelschicht auf dem Erwerbsarbeitsmarkt, sondern eine Ideologie, die im Kern die Naturhaftigkeit gesellschaftlicher Grenzziehungen und Ausschlüsse postuliert.

Foucaults oben zitierte Frage unterstellt, dass nicht unbedingt klar ist, wer ausgeschlossen und zur Ausmerzung vorgesehen ist. Sie rekurriert auf die postkoloniale Kondition, deren Hauptcharakteristika Grenzverwischungen und Vermischungen sind. Koloniale Diskurse definierten klare Grenzen zwischen innen und außen, zwischen Kolonie und Mutterland, zwischen „Rassen“, Geschlechtern und Klassen. Die post-koloniale Kondition wirbelt Grenzen und Räume durcheinander. Die Interventionen ehemals Kolonisierter in den postkolonialen Metropolen des Westens machen dem Traum eines rassisch homogenen deutschen Volkskörpers ein Ende und stellen die selbstverständlich unausgesprochenen Privilegien Weißer deutscher Eliten zur Disposition (vgl. Ha et al. 2007).

Die einzige Lösung, die der Weißen Mittelschicht zusichert, dass das Horrorszenario des Im-Müll-Lebens und Müll-Seins für sie nie Wahrheit wird, ist „Rasse“. Nur wenn die Verwahrlosung schon im Körper angelegt, nur wenn sie unverlierbar in den Körper eingeschrieben ist, können wohlhabende Weiße zwar verarmen, aber nie zum White trash werden. Von dem Vorschlag Oda Olbergs, das Lumpenproletariat als biologische Kategorie $\mathrm{zu}$ fassen, welches es bevölkerungspolitisch $\mathrm{zu}$ dezimieren gälte, sind wir gar nicht so weit entfernt: Auf dem Feld der Genetik entfalten sich ungeahnte Möglichkeiten, die ,genetische Unterschicht“ erst 
zu konstruieren, dann zu denunzieren und schließlich zur Ausmerzung $\mathrm{zu}$ bestimmen. Die neurobiologische Forschung eines Wolf Singer (Frankfurt) und Gerhard Roth (Bremen) beispielsweise spricht von genetischen Prädispositionen, der Genetiker Volkmar Weiß (Leipzig) von der Erblichkeit der Intelligenz. Weiß steht der NPD nahe, jedoch auch im linken Lager existieren Vorstellungen von Gruppen, denen mit bevölkerungspolitischen Maßnahmen zu begegnen sei: Christa Müller, Mitglied im Landesvorstand der Saar-Linkspartei und Ehefrau von Oskar Lafontaine, sagte bei einer Pressekonferenz im Oktober 2006, durch umfassende staatliche Familienberatung lasse sich die „Reproduktion des asozialen Milieus" begrenzen.

Die Imagination eines gesunden deutschen Volkskörpers, der bedrohliche Krankheitskeime in sich trägt, deren Ausbreitung es zu verhindern gilt, ist kein Thema des Geschichtsunterrichts allein. Gegenwärtige Diskurse zum ,abgehängten Prekariat“ revitalisieren zutiefst rassifizierte Bilder der Abgeschriebenen, Abgestumpften, und letztlich bereits Abgestorbenen, die, da verloren für die „Rasse“, zu Objekten eugenischer Maßnahmen werden.

Die Geschichte qualmt noch...

\section{Literatur}

Bublitz, Hannelore (2000): „Sozialdarwinismus als Schnittstelle der Rationalisierung von Arbeit, Bevölkerungspolitik und Sexualität“. In: Hannelore Bublitz/Christine Hanke/Andrea Seier (Hg.), Der Gesellschaftskörper. Zur Neuordnung von Kultur und Geschlecht um 1900, Frankfurt/M.: Campus.

Eggers, Maureen Maisha/Kilomba, Grada/Piesche, Peggy/Arndt, Susan (2005) (Hg.): Mythen, Masken und Subjekte. Kritische Weißseinsforschung in Deutschland, Münster: Unrast.

Foucault, Michel (1976): Mikrophysik der Macht. Über Strafjustiz, Psychiatrie und Macht, Berlin: Merve.

GEO Wissen, 35 (März 2005), Sünde und Moral.

Grosse, Pascal (2000): Kolonialismus, Eugenik und bürgerliche Gesellschaft in Deutschland 1850-1918, Frankfurt/M.: Campus.

Ha, Kien Nghi/al-Samarai, Nicola Lauré/Mysorekar, Sheila (2007) (Hg.): re/visionen. Postkoloniale Perspektiven von People of Color auf Rassismus, Kulturpolitik und Widerstand in Deutschland, Münster: Unrast. 
DGB-Jugend (2007) (Hg.): Generation Praktikum? Prekäre Beschäftigungsformen von Hochschulabsolventinnen und -absolventen, Berlin, http://www.boeckler.de/pdf/fof_praktikum_2007.pdf, 15.03. 2008.

Hund, Wulf D. (1999): Rassismus, Münster: Westfälisches Dampfboot.

Kessel, Fabian (2005): „Das wahre Elend? Zur Rede von der neuen ,Unterschicht““. In: Widersprüche 25 (98), S. 29-42.

Marx, Karl: Klassenkämpfe 1884-1850. MEW 7, Berlin: Dietz.

Marx, Karl: 18. Brumaire. MEW 8, Berlin: Dietz.

Marx, Karl: Kommunistisches Manifest. 1848, MEW 4, Berlin: Dietz.

Müller-Hilmer, Rita (2006): Gesellschaft im Reformprozess, Bonn: Friedrich-Ebert-Stiftung, http://www.fes.de/inhalt/Dokumente/06101 7_Gesellschaft_im_Reformprozess_komplett.pdf, 15.3.2008.

Newitz, Annalee/Wray, Matthew (1997): „What is ,White Trash“"? Stereotypes and Economic Conditions of Poor Whites in the United States“. In: Mike Hill (Hg.), Whiteness. A Critical Reader, New York/London: NYU Press, S. 168-184.

Nolte, Paul (2004): Generation Reform. Jenseits der blockierten Republik, Bonn: Bundeszentrale für politische Bildung.

Nolte, Paul (2006): Riskante Moderne. Die Deutschen und der neue Kapitalismus, München: Bundeszentrale für politische Bildung.

Schmidt-Lauber, Brigitta (1998): „Die verkehrte Hautfarbe“. Ethnizität deutscher Namibier als Alltagspraxis, Berlin/Hamburg: Reimer.

Tutt, Cordula (2007): Das große Schrumpfen, Berlin: Berlin-Verlag.

Wollrad, Eske (2005): Weißsein im Widerspruch. Feministische Perspektiven auf Rassismus, Kultur und Religion, Königstein/Taunus: Helmer. 
